\title{
Practice Patterns in the Acceptance of Medically Complex Living Kidney Donors with Obesity, Hypertension, Family History of Kidney Disease, or Donor-Recipient Age Discrepancy
}

\author{
Ziad Arabi ${ }^{1}$ Muhammad Bukhari2 ${ }^{2}$ Abdullah Hamad ${ }^{3}$ \\ ${ }^{1}$ Department of the Organ Transplant Center, Division of Adult \\ Transplant Nephrology, King Abdulaziz Medical City, King Abdullah \\ International Medical Research Center, King Saud Bin Abdulaziz \\ University for Health Sciences, Riyadh, Saudi Arabia \\ 2 Department of Medicine, Division of Adult Nephrology, Taif \\ University, Taif, Saudi Arabia \\ ${ }^{3}$ Department of Medicine, Division of Nephrology, Regional Medical \\ Center of Orangeburg and Calhoun Counties, Orangeburg, South \\ Carolina, United States \\ ${ }^{4}$ Department of Medicine, Division of Nephrology, CHU, Clermont- \\ Ferrand, France
}

Abdulrahman Altheaby ${ }^{10}$ Saleh Kaysi ${ }^{4}$

Address for correspondence Ziad Arabi, MD, Department of the Organ Transplant Center, Division of Adult Transplant Nephrology, King Abdulaziz Medical City, Riyadh, Saudi Arabia (e-mail: ziadarabi@yahoo.com).

Avicenna J Med 2021;11:172-184.
Abstract
Keywords
- Age
- Diabetes
- Extended criteria
- Family history of kidney disease
- Gender
- Hypertension
- Living kidney donor
- Metabolic syndrome
- Obesity
- Pregnancy
- Survey

Background To assess the practice patterns of the acceptance of medically complex living kidney donors (MCLKDs).

Methods We distributed a survey to nephrologists and transplant surgeons (TS) across the world through major international transplant societies. The survey contained questions regarding obesity, abnormal blood glucose profile, mild hypertension, donor-recipient age discrepancy, or family history of kidney disease of unknown etiology.

Results In total, 239 respondents from 29 countries (42\% were nephrologists and $58 \%$ were TS).

Most respondents would allow donations from obese donors, especially if they intended to lose weight but would be cautious if these donors had abnormal blood glucose or family history of diabetes mellitus. In hypertensive donors, future pregnancy plans mattered in decisions regarding the acceptance of female donors. Most respondents would allow young donors but would be more cautious if they had a future risk of hypertension or a family history of kidney disease of unknown etiology. They would also allow donations from an older person if prolonged waiting time was anticipated. We found multiple areas of consensus of practice among the diverse members of international transplant societies, with some interesting variations among nephrologists and TS.

Conclusions This survey highlights the practice patterns of the acceptance of MCLKDs among the international community. In the absence of clear guidelines, this survey provides additional information to counsel kidney donors with these conditions. published online

November 15, 2021
DOI https://doi.org/ $10.1055 / \mathrm{s}-0041-1736541$ ISSN 2231-0770.

\footnotetext{
(c) 2021. Syrian American Medical Society. All rights reserved. This is an open access article published by Thieme under the terms of the Creative Commons Attribution-NonDerivative-NonCommercial-License, permitting copying and reproduction so long as the original work is given appropriate credit. Contents may not be used for commercial purposes, or adapted, remixed, transformed or built upon. (https://creativecommons.org/ licenses/by-nc-nd/4.0/)

Thieme Medical and Scientific Publishers Pvt. Ltd., A-12, 2nd Floor, Sector 2, Noida-201301 UP, India
} 


\section{Introduction}

Potential living kidney donors (LKDs) are frequently found to have borderline medical or surgical abnormalities, complicating the evaluation of kidney donation. These donors are often referred to as medically complex living kidney donors (MCLKDs). MCLKDs have many medical conditions such as obesity, mildly abnormal glucose profile, mild hypertension, or family history (FH) of kidney disease. ${ }^{1}$ Although the exact prevalence of MCLKDs in an entire pool of donors is unknown, they constitute approximately at least $25 \%$ of LKDs. ${ }^{2}$

Kidney donations carry potential risks long-term risks, especially end-stage kidney disease (ESKD) for donors. ${ }^{3-6}$ These risks are potentially higher in MCLKDs. The guidelines for kidney donation do not adequately address several controversial issues of MCLKDs due to lack of evidence-based data. ${ }^{7-9}$ Similarly, online risk calculators can provide general estimations of risk of ESKD after kidney donation ${ }^{10,11}$ but this does not apply to MCLKDs. The renal functional reserve of donors in the long-term cannot be accurately predicted at the time of donation. ${ }^{12}$ Thus, experts' perspectives on the utility and acceptance of MCLKDs may vary. . $^{13,14}$

This study aimed to assess the practice patterns of the acceptance of MCLKDs with obesity, abnormal glucose profile, hypertension, $\mathrm{FH}$ of kidney disease of unknown etiology and donor-recipient age discrepancy, and to explore the areas of practice where guidelines are most needed.

\section{Materials and Methods}

The survey was based on a thorough literature review of studies on LKDs with complex medical problems, ${ }^{1,2,13-16}$ group discussion with expert transplant specialists (from nephrology and surgical fields), clinical experiences of dealing with these difficult cases, trends of transplant societies, and controversies arising in conferences. Our survey consisted of 31 questions. The first five questions focused on the demographic characteristics of the participants. The first 17 questions of the survey are discussed in this paper, and the remaining 9 questions are discussed in our concurrent submission. The questions discussed in this paper were grouped into four domains, representing the most frequently encountered and challenging issues about suitability and acceptance of MCLKDs: (1) obesity or abnormal glucose profile (8 questions), (2) mild hypertension (3 questions), (3) donor-recipient age discrepancy (4 questions), and (4) donors with FH of kidney disease of unknown origin (2 questions).

The cross-sectional survey was distributed to participants using SurveyMonkey after obtaining approval from the Institutional Review Board (IRB) (RC19/149/R). Six international transplant societies were contacted to distribute the survey to their members, including nephrologists and transplant surgeons (TS). However, only the American Society of Transplantation (AST), the American Society of Transplant Surgeons (ASTS), and the European Renal Association-European Dialysis and Transplant Association (ERA-EDTA) responded. Three transplant societies did not participate due to not responding.
The survey was distributed between June 2019 and February 2020 by the participating societies according to their respective policies. (AST: through the society email and directly to the members with five subsequent reminders, ERA-EDTA: by publishing the survey on its Web site, and ASTS: by using the "survey rental" of the society to email the questionnaires to the members with five subsequent reminders).

The inclusion criteria were as follows: practicing nephrologists and TS. In training or nonpracticing nephrologists or TS were requested not to participate. After obtaining informed consent online, participants were requested to choose the best option for the given scenarios. The frequency of the total responses to the questions was reported as the number and percentage of the total number of participants, nephrologists, and TS. SPSS (version 24) was used for data analysis. Comparisons were performed using $\mathrm{X} 2$ test or Fisher's exact test as appropriate. Two-sided $p$-values of less than 0.05 were defined as statistically significant.

\section{Results}

\section{Demographic Characteristics of Participants}

A total of 239 nephrologists and TS from 29 countries responded to the survey; $64 \%(n=154)$ of the respondents were from the United States. Among these, $42 \%(n=100)$ were nephrologists and $58 \%(n=139)$ were TS. Majority of the respondents (77\%) had many years of work experience (> 6 years in practice) and were also heavily involved in pretransplant evaluations of donors and recipients. -Table 1 summarizes the demographic characteristics of the respondents.

Among the respondents, compared with nephrologists, TS had more years of work experience and were more involved in pretransplant evaluations of donors and recipients. Moreover, TS were more likely to be practicing in the United States $(p<0.05)$, as shown in - Table 1.

The response rate of the living donor and kidney and pancreas committee members of AST was 5.5\%. The response rate of ASTS members was 10.4. Due to the nature of the survey distribution method by web posting through EDTAERA, the response rate could not be attained. The total responses from ERA-EDTA constituted 19\% of the entire responses of the survey though. The survey completion rate (the rate at which the respondents completed the survey entirely) was $99 \%$.

\section{Evaluation of Medically Complex Donors}

\section{Obesity or Abnormal Glucose Profile}

From the data analysis, we found that obesity stage I (body mass index [BMI] 30-35) was not considered as a major challenge for kidney donation. Of the respondents, $75 \%$ answered that they would accept obese donors if they showed some weight loss before donation or if they were at least committed to losing weight in the future. In contrast, $76 \%$ felt that donors with obesity stage II (BMI 36-40) would require some weight loss or reduction of BMI to 30 . 
174 Practice Patterns in the Acceptance of Medically Complex Living Kidney Donors Arabi et al.

Table 1 The characteristics of participants

\begin{tabular}{|c|c|c|c|c|c|}
\hline Characteristic & Answers & Nephrologists & TS & $p$-Value & Total \\
\hline & & $\begin{array}{l}100 \\
(41.5 \%)\end{array}$ & $\begin{array}{l}139 \\
(57.7 \%)\end{array}$ & & $\begin{array}{l}239 \\
(100 \%)\end{array}$ \\
\hline \multirow[t]{4}{*}{ Practice } & $<1$ year & $\begin{array}{ll}3 \\
3 \%\end{array}$ & $\begin{array}{l}2 \\
1.4 \%\end{array}$ & 0.022 & $\begin{array}{l}5 \\
2.1 \%\end{array}$ \\
\hline & $1-5$ years & $\begin{array}{l}29 \\
29 \%\end{array}$ & $\begin{array}{l}19 \\
13.7 \%\end{array}$ & & $\begin{array}{l}48 \\
20.1 \%\end{array}$ \\
\hline & $6-10$ years & $\begin{array}{l}14 \\
14 \%\end{array}$ & $\begin{array}{l}24 \\
17.3 \%\end{array}$ & & $\begin{array}{l}38 \\
15.9 \%\end{array}$ \\
\hline & More than 10 years. & $\begin{array}{l}54 \\
54 . \%\end{array}$ & $\begin{array}{l}94 \\
67.6 \%\end{array}$ & & $\begin{array}{l}148 \\
61.9 \%\end{array}$ \\
\hline \multirow{4}{*}{$\begin{array}{l}\text { Do you assess } \\
\text { donors for } \\
\text { pretransplant } \\
\text { workup? }\end{array}$} & On a weekly basis. & $\begin{array}{l}50 \\
50 \%\end{array}$ & $\begin{array}{l}77 \\
55.4 \%\end{array}$ & 0.006 & $\begin{array}{l}127 \\
53.1 \%\end{array}$ \\
\hline & On a monthly basis. & $\begin{array}{l}15 \\
15 \%\end{array}$ & $\begin{array}{l}36 \\
25.9 \%\end{array}$ & & $\begin{array}{l}51 \\
21.3 \%\end{array}$ \\
\hline & Not on a regular basis. & $\begin{array}{l}21 \\
21 \%\end{array}$ & $\begin{array}{l}21 \\
15.1 \%\end{array}$ & & $\begin{array}{l}42 \\
17.6 \%\end{array}$ \\
\hline & I do not do donor assessment. & $\begin{array}{l}14 \\
14 \%\end{array}$ & $\begin{array}{l}5 \\
3.6 \%\end{array}$ & & $\begin{array}{l}19 \\
7.9 \%\end{array}$ \\
\hline \multirow{5}{*}{$\begin{array}{l}\text { Do you assess } \\
\text { recipients for } \\
\text { pretransplant } \\
\text { workup? }\end{array}$} & On a weekly basis. & $\begin{array}{l}55 \\
55 \%\end{array}$ & $\begin{array}{l}113 \\
81.9 \%\end{array}$ & $<0.001$ & $\begin{array}{l}168 \\
70.6 \%\end{array}$ \\
\hline & On a monthly basis. & $\begin{array}{l}16 \\
16 \%\end{array}$ & $\begin{array}{l}15 \\
10.9 \% \\
\end{array}$ & & $\begin{array}{l}31 \\
13 \% \\
\end{array}$ \\
\hline & Not on a regular basis. & $\begin{array}{l}11 \\
11 \%\end{array}$ & $\begin{array}{l}8 \\
5.8 \%\end{array}$ & & $\begin{array}{l}19 \\
8 \%\end{array}$ \\
\hline & $\begin{array}{l}\text { I do not do a pretransplant assessment, but I } \\
\text { see postrenal transplant patients. }\end{array}$ & $\begin{array}{l}6 \\
6 \%\end{array}$ & $\begin{array}{l}1 \\
0.7 \%\end{array}$ & & $\begin{array}{l}7 \\
2.9 \%\end{array}$ \\
\hline & $\begin{array}{l}\text { I do not do a pretransplant assessment and I } \\
\text { do not follow renal transplant patients. }\end{array}$ & $\begin{array}{l}12 \\
12 \%\end{array}$ & $\begin{array}{l}1 \\
0.7 \%\end{array}$ & & $\begin{array}{l}13 \\
5.5 \%\end{array}$ \\
\hline \multirow[t]{6}{*}{ Country } & United States & $\begin{array}{l}42 \\
42.4 \%\end{array}$ & $\begin{array}{l}112 \\
81.8 \%\end{array}$ & $<0.001$ & $\begin{array}{l}154 \\
65.3 \%\end{array}$ \\
\hline & Saudi Arabia & $\begin{array}{l}9 \\
9.1 \%\end{array}$ & $\begin{array}{l}4 \\
2.9 \%\end{array}$ & & $\begin{array}{l}13 \\
5.5 \%\end{array}$ \\
\hline & Pakistan & $\begin{array}{l}10 \\
10.1 \%\end{array}$ & $\begin{array}{l}2 \\
1.5 \%\end{array}$ & & $\begin{array}{l}12 \\
5.1 \%\end{array}$ \\
\hline & Sudan & $\begin{array}{l}8 \\
8.1 \%\end{array}$ & $\begin{array}{l}1 \\
0.7 \%\end{array}$ & & $\begin{array}{l}9 \\
3.8 \%\end{array}$ \\
\hline & Canada & $\begin{array}{l}4 \\
4 \%\end{array}$ & $\begin{array}{l}4 \\
2.9 \%\end{array}$ & & $\begin{array}{l}8 \\
3.4 \%\end{array}$ \\
\hline & Other & $\begin{array}{ll}26 \\
26.3 \%\end{array}$ & $\begin{array}{l}14 \\
10.2 \%\end{array}$ & & $\begin{array}{ll}40 \\
15.9 \%\end{array}$ \\
\hline
\end{tabular}

Abbreviation: TS, transplant surgeon.

Moreover, $17 \%$ of the respondents answered that they would decline such donors without considering weight reduction due to the risk of relapse of obesity.

Only $45 \%$ of the respondents would accept obese candidates with a strong FH of diabetes mellitus (DM), including those with normal fasting glucose (FG) and hemoglobin A1C (HgbA1C). Moreover, a significant number of respondents would not accept such candidates unless they lost weight and reduced their BMI to an acceptable level of 30 (32\%) or 25 (7\%). Only $<1 \%$ answered that they would decline an obese donor candidate (BMI if 30-35) with normal glucose profile.
However, the decline rate significantly increased to $16 \%$ for the same donor if they had a strong $\mathrm{FH}$, as obesity is likely to relapse in such a case $(p<0.001)$.

Regarding obese donors with impaired fasting glucose (IFG), $45 \%$ of the respondents would decline the kidney donation. Among the respondents, 32\% would delay kidney donation until BMI is reduced to below 30 with resolution of impaired fasting glucose (IFG) which is secondary to weight reduction. More than half of the respondents (55\%) would not rely solely on isolated mildly elevated $\mathrm{Hgb} \mathrm{A} 1 \mathrm{C}$ or isolated IFG as the critical indicator for declining a young donor with 


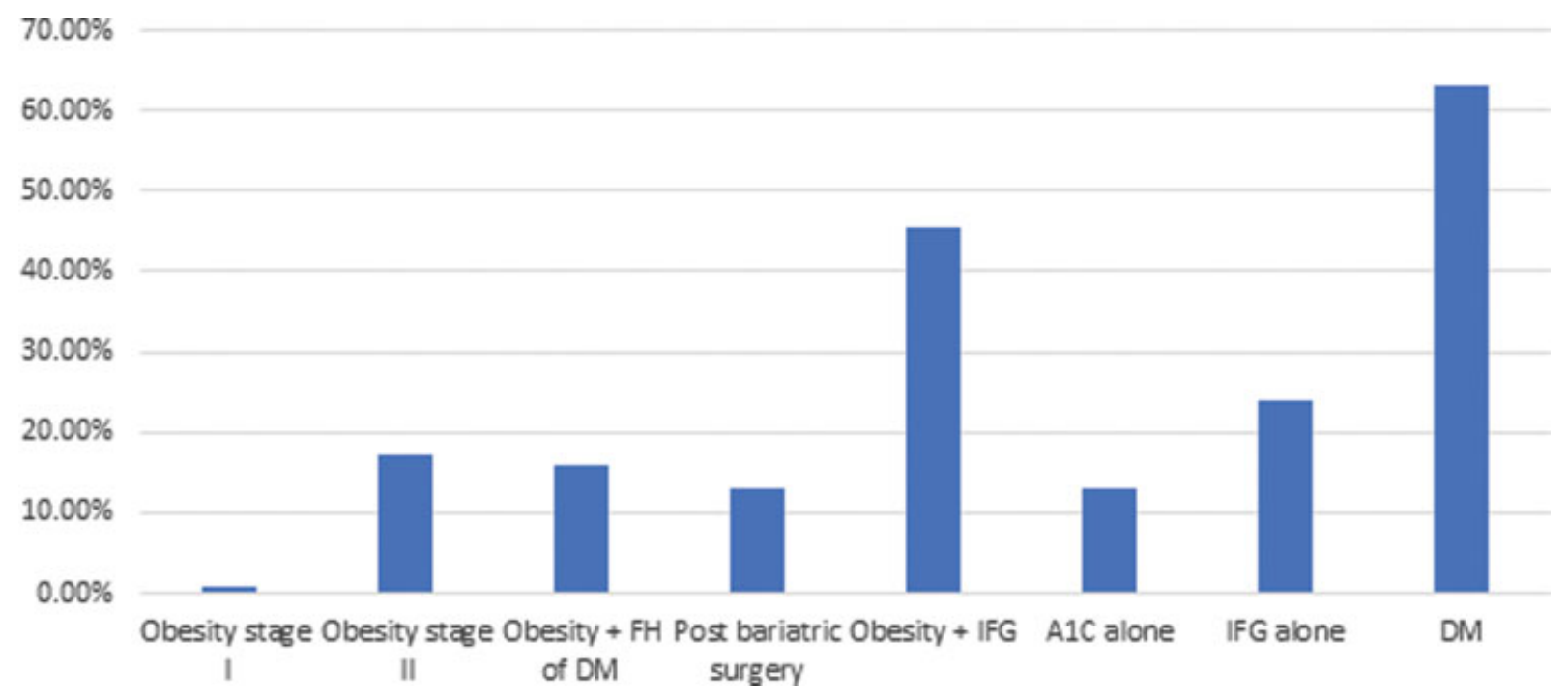

Fig. 1 The decline rate of medically complex donors with obesity or abnormal glucose profile.

normal weight and normal FG, but they would include a glucose tolerance test (GTT) to decide on whether to accept such donors.

Of the respondents, $63 \%$ indicated that the presence of even mild and controlled diabetes in a middle age-old donor was a contraindication for kidney donation. However, such donors might be allowed to donate if no alternative donor was available after counseling them about the risks involved (24\%). Regarding potential donors with resolved DM due to weight loss after bariatric surgery, $36 \%$ of the respondents would consider them for donation if obesity did not relapse 2 years after the surgery. Additionally, 47\% may consider such donors earlier (once BMI is below $30,29 \%$ ), while $18 \%$ may consider them immediately after bariatric surgery.

Isolated obesity stage I (with normal blood profile) was not considered an absolute contraindication for kidney donation (only $<1 \%$ of the respondents would decline such candidates). However, the decline rate increased significantly when other risk factors were included (up to $16 \%$ in the presence of $\mathrm{FH}$ [ $p<0.001], 17 \%$ in obesity stage II [ $p<0.001]$, and $45 \%$ in IFG [ $p<.0001]$ ). Only $13 \%$ of the respondents would immediately decline a young donor based on Hgb A1C alone, but almost twice as many (24.5\%) would rely on IFG alone to decline a similar donor $(p=0.001)$, as indicated in - Fig. 1 .

Compared with nephrologists, TS were more likely to accept obese donor candidates, obese candidates with an FH of DM, or postgastric sleeve $(p<0.05)$. - Table 2 summarizes the survey data regarding donors with obesity or abnormal glucose profile.

\section{Mild Hypertension}

Majority of the respondents ( $n=165,69 \%)$ would allow a multiparous woman with hypertension to donate if she had mild and controlled hypertension (on single blood pressure [BP] medication) and was not planning on having more children. The remaining respondents would either advise against donation unless there is no alternative donor $(n=39$, $16 \%)$ or decline the donation $(n=33,13.9 \%)$. In contrast, more respondents would reject a primiparous woman to donate in the same clinical scenario if she was considering having children ( $n=103,43 \%$ ) or accept her only if there was no alternative donor $(n=74,31 \%)$.

Most respondents would not allow a 20-year-old man to donate with apparent white coat hypertension if his 24-hour ambulatory BP monitoring (ABPM) showed a nondipping nocturnal pattern $(n=130,54,6 \%)$ or they would accept him only if there was no alternative donor ( $n=64,26,9 \%)$. No differences were observed between the opinions of nephrologists and surgeons regarding these three questions. -Table 3 summarizes the survey data regarding donors with hypertension issues.

\section{Donor-Recipient Age Discrepancy}

Most respondents ( $n=170,71.1 \%)$ would allow an 18-yearold man to donate after counseling. However, this percentage decreased $(n=136,57.1 \%)$ if it was an 18-year-old female candidate $(p=0.001)$, which reflects the impact of childbearing as a major concern in considering kidney donation. On the contrary, approximately $20 \%$ of the respondents would decline the donor regardless of the donor's gender. The acceptance rate of an 18-year-old donor dropped significantly to $43 \%(n=102)$ if the recipient was a 75 -year-old. However, in the case of a donor over 65 years, and an 18year-old recipient, with expected prolonged waiting time for a deceased donor, $66 \%(n=157)$ of the respondents would advise finding an alternative donor but allow the donation if no alternative donor was available. Of the respondents, $28 \%$ would decline an 18 -year-old donor for a 75 -year-old recipient but only $9 \%$ would decline 65 -year-old donor for an 18 year-old recipient $(p<0001)$. - Table 4 summarizes the responses to questions regarding donor-recipient age discrepancy. 
Table 2 The acceptance of the MLKD with obesity or abnormal glucose profile

\begin{tabular}{|c|c|c|c|c|c|}
\hline Question & Answer choices & Nephrologist & TS & $p$-Value & Total \\
\hline \multirow{5}{*}{$\begin{array}{l}\text { Q1. The donor is } \\
\text { obese (BMI 30- } \\
\text { 35) with } \\
\text { normal (fasting } \\
\text { blood sugar } \\
\text { and } \\
\text { hemoglobin } \\
\text { A1C) and no } \\
\text { family history } \\
\text { of diabetes): }\end{array}$} & $\begin{array}{l}\text { I will allow this donor to donate now } \\
\text { if the donor is intending to lose } \\
\text { weight later. }\end{array}$ & $\begin{array}{l}34 \\
34.00 \%\end{array}$ & $\begin{array}{l}74 \\
53.60 \%\end{array}$ & \multirow[t]{5}{*}{0.030} & $\begin{array}{l}108 \\
45.4 \%\end{array}$ \\
\hline & $\begin{array}{l}\text { I will allow this donor to donate } \\
\text { once he/she demonstrates some } \\
\text { weight loss. }\end{array}$ & $\begin{array}{l}38 \\
38.00 \%\end{array}$ & $\begin{array}{l}34 \\
24.6 \%\end{array}$ & & $\begin{array}{l}72 \\
30.3 \%\end{array}$ \\
\hline & $\begin{array}{l}\text { I will NOT consider this donor to } \\
\text { donate until he achieves a BMI: } 30 \text {. }\end{array}$ & $\begin{array}{l}22 \\
22.0 \%\end{array}$ & $\begin{array}{l}25 \\
18.1 \%\end{array}$ & & $\begin{array}{l}47 \\
19.7 \%\end{array}$ \\
\hline & $\begin{array}{l}\text { I will NOT consider this donor to } \\
\text { donate until he achieves a BMI: } 25 \text {. }\end{array}$ & $\begin{array}{l}5 \\
5.0 \%\end{array}$ & $\begin{array}{l}4 \\
2.9 \%\end{array}$ & & $\begin{array}{l}9 \\
3.8 \%\end{array}$ \\
\hline & $\begin{array}{l}\text { I will deny this donor because } \\
\text { obesity is a chronic problem and } \\
\text { relapse is likely. }\end{array}$ & $\begin{array}{l}1 \\
1.0 \%\end{array}$ & $\begin{array}{l}1 \\
0.7 \%\end{array}$ & & $\begin{array}{l}2 \\
0.8 \%\end{array}$ \\
\hline \multirow{5}{*}{$\begin{array}{l}\text { Q2. The donor is } \\
\text { obese and has } \\
\text { a strong family } \\
\text { history of } \\
\text { diabetes (but } \\
\text { normal fasting } \\
\text { blood sugar } \\
\text { and } \\
\text { hemoglobin } \\
\text { A1C): }\end{array}$} & $\begin{array}{l}\text { I will allow this donor to donate now } \\
\text { if the donor is intending to lose } \\
\text { weight later. }\end{array}$ & $\begin{array}{l}10 \\
10.0 \%\end{array}$ & $\begin{array}{l}35 \\
25.4 \%\end{array}$ & \multirow[t]{5}{*}{0.004} & $\begin{array}{l}45 \\
18.9 \%\end{array}$ \\
\hline & $\begin{array}{l}\text { I will allow this donor to donate } \\
\text { once he/she demonstrates some } \\
\text { weight loss. }\end{array}$ & $\begin{array}{l}21 \\
21.0 \%\end{array}$ & $\begin{array}{l}41 \\
29.7 \%\end{array}$ & & $\begin{array}{l}62 \\
26.1 \%\end{array}$ \\
\hline & $\begin{array}{l}\text { I will NOT consider this donor to } \\
\text { donate until he achieves a BMI: } 30 \text {. }\end{array}$ & $\begin{array}{l}40 \\
40.0 \%\end{array}$ & $\begin{array}{l}36 \\
26.1 \%\end{array}$ & & $\begin{array}{l}76 \\
31.9 \%\end{array}$ \\
\hline & $\begin{array}{l}\text { I will NOT consider this donor to } \\
\text { donate until he achieves a BMI: } 25 \text {. }\end{array}$ & $\begin{array}{l}10 \\
10.0 \%\end{array}$ & $\begin{array}{l}7 \\
5.1 \%\end{array}$ & & $\begin{array}{l}17 \\
7.1 \%\end{array}$ \\
\hline & $\begin{array}{l}\text { I will deny this donor because } \\
\text { obesity is a chronic problem and } \\
\text { obese donors tend to regain weight } \\
\text { after donation. }\end{array}$ & $\begin{array}{l}19 \\
19.0 \%\end{array}$ & $\begin{array}{l}19 \\
13.8 \%\end{array}$ & & $\begin{array}{l}38 \\
16.0 \%\end{array}$ \\
\hline \multirow{5}{*}{$\begin{array}{l}\text { Q3. The donor is } \\
\text { obese stage } 2 \\
\text { (BMI 36-40) } \\
\text { with normal } \\
\text { fasting blood } \\
\text { sugar, } \\
\text { hemoglobin } \\
\text { A1C and } \\
\text { Negative } \\
\text { family history } \\
\text { of diabetes }\end{array}$} & $\begin{array}{l}\text { I will allow this donor to donate now } \\
\text { if the donor is intending to lose } \\
\text { weight later. }\end{array}$ & $2.0 \%$ & $\begin{array}{l}8 \\
5.8 \%\end{array}$ & \multirow[t]{5}{*}{0.020} & $\begin{array}{l}10 \\
4.2 \%\end{array}$ \\
\hline & $\begin{array}{l}\text { I will allow this donor to donate } \\
\text { once he/ she demonstrates some } \\
\text { weight loss. }\end{array}$ & $\begin{array}{l}24 \\
24.0 \%\end{array}$ & $\begin{array}{l}55 \\
39.9 \%\end{array}$ & & $\begin{array}{l}79 \\
33.2 \%\end{array}$ \\
\hline & $\begin{array}{l}\text { I will NOT consider this donor to } \\
\text { donate until he achieves a BMI: } 30 \text {. }\end{array}$ & $\begin{array}{l}49 \\
49.0 \%\end{array}$ & $\begin{array}{l}53 \\
38.4 \%\end{array}$ & & $\begin{array}{l}102 \\
42.9 \%\end{array}$ \\
\hline & $\begin{array}{l}\text { I will NOT consider this donor to } \\
\text { donate until he achieves a BMI: } 25 \text {. }\end{array}$ & $\begin{array}{l}2 \\
2.0 \%\end{array}$ & $\begin{array}{l}4 \\
2.9 \%\end{array}$ & & $\begin{array}{l}6 \\
2.5 \%\end{array}$ \\
\hline & $\begin{array}{l}\text { I will decline this donor because } \\
\text { obesity is a chronic problem and } \\
\text { obese donors tend to regain weight } \\
\text { after donation. }\end{array}$ & $\begin{array}{l}23 \\
23.0 \%\end{array}$ & $\begin{array}{l}18 \\
13.0 \%\end{array}$ & & $\begin{array}{l}41 \\
17.2 \%\end{array}$ \\
\hline \multirow{4}{*}{$\begin{array}{l}\text { Q4. The donor is } \\
\text { obese and has } \\
\text { impaired } \\
\text { fasting blood } \\
\text { sugar: }\end{array}$} & $\begin{array}{l}\text { I will allow this donor to donate now } \\
\text { but the donor needs to lose weight } \\
\text { later and to follow his/her blood } \\
\text { sugar. }\end{array}$ & $\begin{array}{l}1 \\
1.0 \%\end{array}$ & $\begin{array}{l}1 \\
0.7 \%\end{array}$ & \multirow[t]{4}{*}{0.225} & $\begin{array}{l}2 \\
0.8 \%\end{array}$ \\
\hline & $\begin{array}{l}\text { I will allow this donor to donate } \\
\text { once impaired BS is resolved due to } \\
\text { some weight reduction. }\end{array}$ & $\begin{array}{l}11 \\
11.1 \%\end{array}$ & $\begin{array}{l}29 \\
21.0 \%\end{array}$ & & $\begin{array}{l}40 \\
16.9 \%\end{array}$ \\
\hline & $\begin{array}{l}\text { I will allow this donor to donate } \\
\text { once impaired BS is resolved and } \\
\text { BMI is below } 30 \text { due to weight } \\
\text { reduction. }\end{array}$ & $\begin{array}{l}31 \\
31.3 \%\end{array}$ & $\begin{array}{l}45 \\
32.6 \%\end{array}$ & & $\begin{array}{l}76 \\
32.1 \%\end{array}$ \\
\hline & $\begin{array}{l}\text { I will allow this donor to donate } \\
\text { once impaired BS is resolved and }\end{array}$ & $\begin{array}{l}6 \\
6.1 \%\end{array}$ & $\begin{array}{l}5 \\
3.6 \%\end{array}$ & & $\begin{array}{l}11 \\
4.6 \%\end{array}$ \\
\hline
\end{tabular}


Table 2 (Continued)

\begin{tabular}{|c|c|c|c|c|c|}
\hline Question & Answer choices & Nephrologist & TS & $p$-Value & Total \\
\hline & $\begin{array}{l}\mathrm{BMI} \text { is below } 25 \text { due to weight } \\
\text { reduction. }\end{array}$ & & & & \\
\hline & $\begin{array}{l}\text { I will decline this donor because this } \\
\text { donor is at high risk to develop } \\
\text { diabetes. }\end{array}$ & $\begin{array}{l}50 \\
50.5 \%\end{array}$ & $\begin{array}{l}58 \\
42.0 \%\end{array}$ & & $\begin{array}{l}108 \\
45.6 \%\end{array}$ \\
\hline \multirow{4}{*}{$\begin{array}{l}\text { Q5. A young donor } \\
\text { with normal } \\
\text { weight and } \\
\text { normal fasting } \\
\text { blood sugar } \\
\text { but mildly } \\
\text { elevated } \\
\text { HbA1C (e.g., } \\
\text { HbA1C = 5.7- } \\
\text { 6) }\end{array}$} & $\begin{array}{l}\text { I will allow this donor to donate now } \\
\text { after giving him/her the } \\
\text { appropriate advice to manage } \\
\text { prediabetes. }\end{array}$ & $\begin{array}{l}13 \\
13.1 \%\end{array}$ & $\begin{array}{l}20 \\
14.4 \%\end{array}$ & \multirow[t]{4}{*}{0.264} & $\begin{array}{l}33 \\
13.9 \%\end{array}$ \\
\hline & $\begin{array}{l}\text { I will repeat } \mathrm{A} 1 \mathrm{C} \text { in } 3 \text { months and I } \\
\text { will decline the donor if still } \\
\text { elevated. }\end{array}$ & $\begin{array}{l}16 \\
16.2 \%\end{array}$ & $\begin{array}{l}25 \\
18 \%\end{array}$ & & $\begin{array}{l}41 \\
17.2 \%\end{array}$ \\
\hline & $\begin{array}{l}\text { I will ask for } 2 \text { hour. GTT and I'll } \\
\text { decline the donor if both GTT and } \\
\text { HbA1C are elevated. }\end{array}$ & $\begin{array}{l}52 \\
52.5 \%\end{array}$ & $\begin{array}{l}81 \\
58.3 \%\end{array}$ & & $\begin{array}{l}133 \\
55.9 \%\end{array}$ \\
\hline & $\begin{array}{l}\text { I will decline this donor donate due } \\
\text { to his/her } A 1 C \text { and young age. }\end{array}$ & $\begin{array}{l}18 \\
18.2 \%\end{array}$ & $\begin{array}{l}13 \\
9.4 \%\end{array}$ & & $\begin{array}{l}31 \\
13.0 \%\end{array}$ \\
\hline \multirow{4}{*}{$\begin{array}{l}\text { Q6. A young donor } \\
\text { with normal } \\
\text { weight and } \\
\text { normal HbA1C } \\
\text { but impaired } \\
\text { fasting blood } \\
\text { sugar }\end{array}$} & $\begin{array}{l}\text { I will allow this donor to donate now } \\
\text { after giving him/her the } \\
\text { appropriate advice to manage } \\
\text { prediabetes. }\end{array}$ & $\begin{array}{l}13 \\
13.3 \%\end{array}$ & $\begin{array}{l}12 \\
8.6 \%\end{array}$ & \multirow[t]{4}{*}{0.066} & $\begin{array}{l}25 \\
10.5 \%\end{array}$ \\
\hline & $\begin{array}{l}\text { I will repeat } A 1 C \text { in } 3 \text { months and I } \\
\text { will decline the donor if still } \\
\text { elevated. }\end{array}$ & $8.2 \%$ & $\begin{array}{l}14 \\
10.1 \%\end{array}$ & & $\begin{array}{l}22 \\
9.3 \%\end{array}$ \\
\hline & $\begin{array}{l}\text { I will ask for } 2 \text { hour. GTT and I'll } \\
\text { decline the donor if both GTT and } \\
\text { HbA1C are elevated. }\end{array}$ & $\begin{array}{l}46 \\
46.9 \%\end{array}$ & $\begin{array}{l}86 \\
61.9 \%\end{array}$ & & $\begin{array}{l}132 \\
55.7 \%\end{array}$ \\
\hline & $\begin{array}{l}\text { I will decline this donor donate due } \\
\text { to his/her impaired fasting sugar } \\
\text { and young age. }\end{array}$ & $\begin{array}{l}31 \\
31.6 \%\end{array}$ & $\begin{array}{l}27 \\
19.4 \%\end{array}$ & & $\begin{array}{l}58 \\
24.5 \%\end{array}$ \\
\hline \multirow{4}{*}{$\begin{array}{l}\text { Q7. A middle age } \\
\text { (e.g., } 55 \text { years } \\
\text { old) donor } \\
\text { with diabetes } \\
\text { for one year } \\
\text { and controlled } \\
\text { with } \\
\text { monotherapy } \\
\text { and diet. }\end{array}$} & $\begin{array}{l}\text { I will allow this donor to donate } \\
\text { now. }\end{array}$ & $\begin{array}{l}6 \\
6.0 \%\end{array}$ & $\begin{array}{l}9 \\
6.5 \%\end{array}$ & \multirow[t]{4}{*}{0.629} & $\begin{array}{l}15 \\
6.3 \%\end{array}$ \\
\hline & $\begin{array}{l}\text { I will allow this donor to donate if } \\
\text { his diabetes gets controlled without } \\
\text { any medication (with diet only). }\end{array}$ & $\begin{array}{l}8 \\
8.0 \%\end{array}$ & $\begin{array}{l}6 \\
4.3 \%\end{array}$ & & $\begin{array}{l}14 \\
5.9 \%\end{array}$ \\
\hline & $\begin{array}{l}\text { I will advise him against donation, } \\
\text { but I may allow him/her to donate if } \\
\text { no alternative donor is available. }\end{array}$ & $\begin{array}{l}22 \\
22.0 \%\end{array}$ & $\begin{array}{l}36 \\
25.9 \%\end{array}$ & & $\begin{array}{l}58 \\
24.3 \%\end{array}$ \\
\hline & $\begin{array}{l}\text { I will not clear this donor to donate } \\
\text { even if there is no alternative living- } \\
\text { donor is available. }\end{array}$ & $\begin{array}{l}64 \\
64.0 \%\end{array}$ & $\begin{array}{l}88 \\
63.3 \%\end{array}$ & & $\begin{array}{l}152 \\
63.6 \%\end{array}$ \\
\hline \multirow{5}{*}{$\begin{array}{l}\text { Q8. The donor is } \\
\text { postgastric } \\
\text { sleeve due to } \\
\text { stage II obesity } \\
\text { (BMI 36-40) } \\
\text { and his } \\
\text { diabetes has } \\
\text { resolved with } \\
\text { weight loss: }\end{array}$} & $\begin{array}{l}\text { I will accept this donor for } \\
\text { immediate donation (once } \\
\text { surgically doable). }\end{array}$ & $99 \%$ & $\begin{array}{l}34 \\
24.5 \%\end{array}$ & \multirow[t]{5}{*}{0.001} & $\begin{array}{l}43 \\
18.0 \%\end{array}$ \\
\hline & $\begin{array}{l}\text { I will consider this donor for } \\
\text { donation once BMI is below } 30 \text {. }\end{array}$ & $\begin{array}{l}24 \\
24.0 \%\end{array}$ & $\begin{array}{ll}46 \\
33.1 \%\end{array}$ & & $\begin{array}{l}70 \\
29.3 \%\end{array}$ \\
\hline & $\begin{array}{l}\text { I will consider this donor for } \\
\text { donation once BMI is down to } 25 \text {. }\end{array}$ & $\begin{array}{l}6 \\
6.0 \%\end{array}$ & $\begin{array}{l}1 \\
0.7 \%\end{array}$ & & $\begin{array}{l}7 \\
2.9 \%\end{array}$ \\
\hline & $\begin{array}{l}\text { I will consider this donor for } \\
\text { donation if two years passed after } \\
\text { bariatric surgery without obesity } \\
\text { relapse. }\end{array}$ & $\begin{array}{l}44 \\
44.0 \%\end{array}$ & $\begin{array}{l}43 \\
30.9 \%\end{array}$ & & $\begin{array}{l}87 \\
36.4 \%\end{array}$ \\
\hline & $\begin{array}{l}\text { I will not consider this donor for } \\
\text { donation. }\end{array}$ & $\begin{array}{l}17 \\
17.0 \%\end{array}$ & $\begin{array}{l}15 \\
10.8 \%\end{array}$ & & $\begin{array}{l}32 \\
13.4 \%\end{array}$ \\
\hline
\end{tabular}

Abbreviations: BMI, body mass index; BS, blood sugar; GTT, glucose tolerance test; MCLKD, medically complex living kidney donors; TS, transplant surgeon. 
Table 3 The acceptance of MCKLD with mild hypertension

\begin{tabular}{|c|c|c|c|c|c|}
\hline Question & Answer choices & Nephrologists & TS & p-Value & Total \\
\hline \multirow{3}{*}{$\begin{array}{l}\text { Q9. The donor is } \\
\text { multiparous women } \\
\text { with mild and } \\
\text { controlled } \\
\text { hypertension (only } \\
\text { on one BP } \\
\text { medication). She is } \\
\text { NOT planning to have } \\
\text { more children. }\end{array}$} & $\begin{array}{l}\text { I allow her to donate after } \\
\text { counselling her about the future } \\
\text { risk after donation. }\end{array}$ & $\begin{array}{l}69 \\
69.0 \%\end{array}$ & $\begin{array}{l}96 \\
70.1 \%\end{array}$ & \multirow[t]{3}{*}{0.302} & $\begin{array}{l}165 \\
69.6 \%\end{array}$ \\
\hline & $\begin{array}{l}\text { I will advise her not to donate but I } \\
\text { will allow the donation if no } \\
\text { alternative donor is available. }\end{array}$ & $\begin{array}{l}20 \\
20.0 \%\end{array}$ & $\begin{array}{l}19 \\
13.9 \%\end{array}$ & & $\begin{array}{l}39 \\
16.5 \%\end{array}$ \\
\hline & I will DECLINE this donor. & $\begin{array}{l}11 \\
11.0 \%\end{array}$ & $\begin{array}{l}22 \\
16.1 \%\end{array}$ & & $\begin{array}{l}33 \\
13.9 \%\end{array}$ \\
\hline \multirow{3}{*}{$\begin{array}{l}\text { Q10. The donor is } \\
\text { primiparous } \\
\text { women with mild } \\
\text { and controlled } \\
\text { hypertension (only } \\
\text { one BP medication). } \\
\text { She is considering } \\
\text { having children } \\
\text { later. }\end{array}$} & $\begin{array}{l}\text { I allow her to donate after } \\
\text { counselling her about the future } \\
\text { risk after donation. }\end{array}$ & $\begin{array}{l}21 \\
21.2 \%\end{array}$ & $\begin{array}{l}40 \\
28.8 \%\end{array}$ & \multirow[t]{3}{*}{0.417} & $\begin{array}{l}61 \\
25.6 \%\end{array}$ \\
\hline & $\begin{array}{l}\text { I will advise her not to donate but I } \\
\text { will allow the donation if no } \\
\text { alternative donor is available. }\end{array}$ & $\begin{array}{l}33 \\
33.3 \%\end{array}$ & $\begin{array}{l}41 \\
29.5 \%\end{array}$ & & $\begin{array}{l}74 \\
31.1 \%\end{array}$ \\
\hline & I will DECLINE this donor. & $\begin{array}{l}45 \\
45.5 \%\end{array}$ & $\begin{array}{l}58 \\
41.7 \%\end{array}$ & & $\begin{array}{l}103 \\
43.3 \%\end{array}$ \\
\hline \multirow{3}{*}{$\begin{array}{l}\text { Q11. A 20-year-old man } \\
\text { with high BP } \\
\text { readings at the } \\
\text { office and a non- } \\
\text { dipping pattern at } \\
\text { night on his } 24 \text { hour. } \\
\text { ambulatory BP } \\
\text { monitoring which is } \\
\text { otherwise normal. }\end{array}$} & $\begin{array}{l}\text { I allow him to donate after } \\
\text { counselling. }\end{array}$ & $\begin{array}{l}13 \\
13.0 \%\end{array}$ & $\begin{array}{l}31 \\
22.5 \%\end{array}$ & \multirow[t]{3}{*}{0.178} & $\begin{array}{l}44 \\
18.5 \%\end{array}$ \\
\hline & $\begin{array}{l}\text { I will advise him not to donate. } \\
\text { However, I will allow the donation if } \\
\text { no alternative donor is available. }\end{array}$ & $\begin{array}{l}29 \\
29.0 \%\end{array}$ & $\begin{array}{l}35 \\
25.4 \%\end{array}$ & & $\begin{array}{l}64 \\
26.9 \%\end{array}$ \\
\hline & I will DECLINE this donor. & $\begin{array}{l}58 \\
58.0 \%\end{array}$ & $\begin{array}{l}72 \\
52.2 \%\end{array}$ & & $\begin{array}{l}130 \\
54.6 \%\end{array}$ \\
\hline
\end{tabular}

Abbreviations: BP, blood pressure; MCLKD, medically complex living kidney donors; TS, transplant surgeon.

Family History of Kidney Disease of Unknown Etiology Almost half of the respondents $(n=122,47 \%)$ would not accept a young donor with a strong FH of kidney disease of unknown etiology despite negative evaluation. The rate of approval increased slightly when there was no alternative donor and the donor insisted on donating due to being a closer relative (e.g., mother) ( $n=125,53 \%$ to $n=157,67 \%$; $p=0.004)$. The difference in the views of nephrologists and TS were not statistically significant. - Table 5 summarizes the responses to questions regarding donors with an FH of kidney disease of unknown etiology.

\section{Discussion}

Evaluating LKDs with obesity is challenging. Obesity is commonly divided into the following three categories: stage I (BMI of 30 to $<35$ ), stage II $(35$ to $<40)$, and stage III $(\geq 40){ }^{17,18}$ Obesity is a major risk factor for type $2 \mathrm{DM}$ and having a higher BMI increases this risk. ${ }^{19,20}$ Additionally, obese patients are at an increased risk of developing proteinuria after unilateral nephrectomy. ${ }^{21}$ For obesity stages I, II, and III, the adjusted relative risk for ESKD is increased to 3, 6 , and 7, respectively, compared with people who had normal weight $(\mathrm{BMI}<25))^{22}$ In contrast, studies showed that MCLKDs with obesity exhibited a similar compensatory increase in function and volume of the remaining kidney compared with nonobese donors 5 years after the dona- tion $^{23}$; obese donors are not at increased risk for long-term reduced renal function compared with nonobese donors with a mean follow-up of 11 years. ${ }^{24}$ These findings support the current practice of using otherwise healthy obese donors while emphasizing the need for more intensive preoperative and postoperative weight loss in such donors. Potential donors with obesity should be counseled about the higher risk of developing diabetes and consequential diabetic kidney disease. ${ }^{25}$ Potential donors with $\mathrm{FH}$ of diabetes are at a higher risk of developing diabetes. The risk is even higher if their parent had DM at a younger age ( $<50$ years; hazard ratio [HR]: 4.7$){ }^{26}$ In our survey, the majority (75\%) of respondents would allow a potential obese donor (BMI 3035) to donate if they started to demonstrate some weight loss or intention to lose weight. However, only $45 \%$ of the respondents would allow a potential obese donor with a strong FH of DM to donate without further requirements or an intention to lose weight. These findings are comparable to those of one survey conducted in the United States in 2017. ${ }^{27}$

Donor nephrectomy in obese donors (stage II or more) is surgically challenging, with longer operation time, more wound complications, and higher risk of delayed graft function (odds ratio [OR]: $2.16, p=0.01$ ). ${ }^{28}$ However, additional serious complications are infrequent, especially at centers with higher annual transplant volume. ${ }^{29}$ The risk of graft failure or recipient mortality is similar across donor BMI groups at the 6th or 11 th month follow-up. ${ }^{28,30,31}$ In our 
Table 4 The acceptance of the MCLKD with regard to age discrepancy

\begin{tabular}{|c|c|c|c|c|c|}
\hline Question & Answer choices & Nephrologists & TS & $p$-Value & Total \\
\hline \multirow{3}{*}{$\begin{array}{l}\text { Q12. Just turned 18-year- } \\
\text { old man who wants } \\
\text { to donate his older } \\
\text { sibling: }\end{array}$} & $\begin{array}{l}\text { I allow him to donate after counselling him } \\
\text { about the future risk after donation. }\end{array}$ & $\begin{array}{l}72 \\
72.0 \%\end{array}$ & $\begin{array}{l}98 \\
70.5 \%\end{array}$ & \multirow[t]{3}{*}{0.668} & $\begin{array}{l}170 \\
71.1 \%\end{array}$ \\
\hline & $\begin{array}{l}\text { I will advise him not to donate because of } \\
\text { his young age and the future risks of CKD } \\
\text { and ESKD. }\end{array}$ & $\begin{array}{l}11 \\
11.0 \%\end{array}$ & $\begin{array}{l}12 \\
8.6 \%\end{array}$ & & $\begin{array}{l}23 \\
9.6 \%\end{array}$ \\
\hline & $\begin{array}{l}\text { I will not consider him for donation at this } \\
\text { age. }\end{array}$ & $\begin{array}{l}17 \\
17.0 \%\end{array}$ & $\begin{array}{l}29 \\
20.9 \%\end{array}$ & & $\begin{array}{l}46 \\
19.2 \%\end{array}$ \\
\hline \multirow{3}{*}{$\begin{array}{l}\text { Q13. Just turned 18-year- } \\
\text { old woman wants to } \\
\text { donate her older } \\
\text { sibling: }\end{array}$} & $\begin{array}{l}\text { I will accept her for donation after } \\
\text { counselling her about the increased risks } \\
\text { of preeclampsia after donation. }\end{array}$ & $\begin{array}{l}56 \\
56.6 \%\end{array}$ & $\begin{array}{l}80 \\
57.6 \%\end{array}$ & \multirow[t]{3}{*}{0.768} & $\begin{array}{l}136 \\
57.1 \%\end{array}$ \\
\hline & $\begin{array}{l}\text { I will advise her not to donate because of } \\
\text { the increased risk of preeclampsia but I } \\
\text { will allow her to donate if she insists and } \\
\text { accepts the risks. }\end{array}$ & $\begin{array}{l}22 \\
22.2 \%\end{array}$ & $\begin{array}{l}26 \\
18.7 \%\end{array}$ & & $\begin{array}{l}48 \\
20.2 \%\end{array}$ \\
\hline & $\begin{array}{l}\text { I will not consider her for donation at this } \\
\text { age. }\end{array}$ & $\begin{array}{l}21 \\
21.2 \%\end{array}$ & $\begin{array}{l}33 \\
23.7 \%\end{array}$ & & $\begin{array}{l}54 \\
22.7 \%\end{array}$ \\
\hline \multirow{3}{*}{$\begin{array}{l}\text { Q14. 18-year-old man } \\
\text { wants to donate to } \\
\text { his } 75 \text {-year-old } \\
\text { father. }\end{array}$} & $\begin{array}{l}\text { I allow him to donate after counselling him } \\
\text { about the future risk after donation. }\end{array}$ & $\begin{array}{l}35 \\
35.4 \%\end{array}$ & $\begin{array}{l}67 \\
48.6 \%\end{array}$ & \multirow[t]{3}{*}{0.081} & $\begin{array}{l}102 \\
43.0 \%\end{array}$ \\
\hline & $\begin{array}{l}\text { I will advise him not to donate because of } \\
\text { the CKD risk and since the recipient might } \\
\text { not get the mortality benefit given his age. }\end{array}$ & $\begin{array}{l}35 \\
35.4 \%\end{array}$ & $\begin{array}{l}33 \\
23.9 \%\end{array}$ & & $\begin{array}{l}68 \\
28.7 \%\end{array}$ \\
\hline & $\begin{array}{l}\text { I will not consider him for donation under } \\
\text { the current age and circumstances. }\end{array}$ & $\begin{array}{l}29 \\
29.3 \%\end{array}$ & $\begin{array}{l}38 \\
27.5 \%\end{array}$ & & $\begin{array}{l}67 \\
28.3 \%\end{array}$ \\
\hline \multirow{3}{*}{$\begin{array}{l}\text { Q15. Donor }>65 \text {-year-old } \\
\text { man to } 18 \text {-year-old } \\
\text { recipient and } \\
\text { expected prolonged } \\
\text { waiting time for } \\
\text { deceased donor }\end{array}$} & I allow him to donate after counselling. & $\begin{array}{l}24 \\
24.0 \%\end{array}$ & $\begin{array}{l}35 \\
25.4 \%\end{array}$ & \multirow[t]{3}{*}{0.960} & $\begin{array}{l}59 \\
24.8 \%\end{array}$ \\
\hline & $\begin{array}{l}\text { I will advise him to find a younger donor, } \\
\text { but I will allow the donation if no } \\
\text { alternative donor is available. }\end{array}$ & $\begin{array}{l}67 \\
67.0 \%\end{array}$ & $\begin{array}{l}90 \\
65.2 \%\end{array}$ & & $\begin{array}{l}157 \\
66.0 \%\end{array}$ \\
\hline & $\begin{array}{l}\text { I will not consider him for donation at this } \\
\text { age. }\end{array}$ & $\begin{array}{l}9 \\
9.0 \%\end{array}$ & $\begin{array}{l}13 \\
9.4 \%\end{array}$ & & $\begin{array}{l}22 \\
9.2 \%\end{array}$ \\
\hline
\end{tabular}

Abbreviations: CKD, chronic kidney disease; ESKD, end-stage kidney disease; MCLKD, medically complex living kidney donors; TS, transplant surgeon.

survey, $62 \%$ of the respondents would decline LKDs with obesity stage II (unchanged rate from a United States survey in 2007). ${ }^{32}$

Donors would be considered at "increased risk for diabetes" or to have "prediabetes" if they meet any of the following three criteria: IFG-100 to $125 \mathrm{mg} / \mathrm{dl}$ (5.6-6.9 mmol/l), impaired oral glucose tolerance-140 to $199 \mathrm{mg} / \mathrm{dl}$ (7.8-11.0 $\mathrm{mmol} / \mathrm{l}$ ), or A1C 5.7 to $6.4 \%$. For all three criteria, the risk is continuous, extending below the lower limit of the range and disproportionately increasing at the higher limit of the range. ${ }^{33}$ Many programs consider potential LKDs with prediabetes. However, they individualize the decision based on the donors' health profile regarding the acceptable risk threshold of the transplant program. ${ }^{8,9}$ In our survey, potential donors with obesity and IFG could donate only if IFG was resolved due to weight reduction, according to half of the respondents.

The cutoff for IFG may vary, but $45 \%$ of the respondents stated it is IFG of $>100 \mathrm{mg} / \mathrm{dl}$, which seems unchanged from 2007. ${ }^{32}$
Previous studies examining the use of HbA1C versus glucose cut points for the diagnosis of DM or IFG found that $\mathrm{HbA1C}$ has the least sensitivity for DM diagnosis in comparison to FG and GTT, which has the highest sensitivity. $^{34}$ The concordance rate of the criteria between the methods used to diagnose diabetes is low, ${ }^{35}$ and it is even lower when the diagnosis is "pre-DM." The oral GTT should be performed for the confirmation of DM among patients with IFG who have an HbA1C between $5.6 \%$ and $6.4 \%{ }^{36,37}$ In our survey, more than half of the respondents would request GTT to verify isolated and mildly elevated A1C or IFG in a potential donor before deciding donation.

DM is considered as an absolute contraindication for kidney donation, according to the European Renal Best Practice Guideline 2014. ${ }^{9}$ Kidney Disease: Improving Global Outcomes (KDIGO) 2017 advises to individualize the decision regarding donor candidates with prediabetes or type 2 DM based on their health profile and the acceptable risk threshold of the transplant program. ${ }^{8}$ In our survey, most respondents would decline a middle-age donor candidate 
Table 5 The acceptance of the MCKLD with family history of kidney disease

\begin{tabular}{|c|c|c|c|c|c|}
\hline Question & Answer choices & Nephrologist & TS & $p$-Value & Total \\
\hline \multirow{3}{*}{$\begin{array}{l}\text { Q16. If an 18-year-old wants } \\
\text { to donate to his brother } \\
\text { ( } 40 \text { years old) and with } \\
\text { a family history on two } \\
\text { uncles with unknown } \\
\text { etiology of ESKD at } \\
40 \text { years. Donor } \\
\text { evaluation is normal } \\
\text { otherwise. }\end{array}$} & $\begin{array}{l}\text { I will clear him to can donate after } \\
\text { counselling. }\end{array}$ & $\begin{array}{l}19 \\
19.0 \%\end{array}$ & $\begin{array}{l}42 \\
30.4 \%\end{array}$ & \multirow[t]{3}{*}{0.133} & $\begin{array}{l}61 \\
25.6 \%\end{array}$ \\
\hline & $\begin{array}{l}\text { I will advise him against donation. However, I } \\
\text { will allow the donation if no alternative donor } \\
\text { is available. }\end{array}$ & $\begin{array}{l}29 \\
29.0 \%\end{array}$ & $\begin{array}{l}36 \\
26.1 \%\end{array}$ & & $\begin{array}{l}65 \\
27.3 \%\end{array}$ \\
\hline & I will not clear him. & $\begin{array}{l}52 \\
52.0 \%\end{array}$ & $\begin{array}{l}60 \\
43.5 \%\end{array}$ & & $\begin{array}{l}112 \\
47.1 \%\end{array}$ \\
\hline \multirow{3}{*}{$\begin{array}{l}\text { Q17. An 18-year-old wants to } \\
\text { donate to his mother } \\
40 \text { years and with a } \\
\text { family history on two } \\
\text { uncles with ESKD at } \\
40 \text { years. Donor } \\
\text { evaluation is negative } \\
\text { otherwise. He } \\
\text { understands the risks of } \\
\text { donation, but he insists } \\
\text { to donate to his mother } \\
\text { since he is the only } \\
\text { available donor. }\end{array}$} & $\begin{array}{l}\text { I will clear him to can donate after } \\
\text { counselling. }\end{array}$ & $\begin{array}{l}24 \\
24.0 \%\end{array}$ & $\begin{array}{l}47 \\
33.8 \%\end{array}$ & \multirow[t]{3}{*}{0.211} & $\begin{array}{l}71 \\
29.7 \%\end{array}$ \\
\hline & $\begin{array}{l}\text { I will advise him against donation. However, I } \\
\text { will allow the donation if no alternative donor } \\
\text { is available. }\end{array}$ & $\begin{array}{l}41 \\
41.0 \%\end{array}$ & $\begin{array}{l}45 \\
32.4 \%\end{array}$ & & $\begin{array}{l}86 \\
36.0 \%\end{array}$ \\
\hline & I will not clear him. & $\begin{array}{l}35 \\
35.0 \%\end{array}$ & $\begin{array}{l}47 \\
33.8 \%\end{array}$ & & $\begin{array}{l}82 \\
34.3 \%\end{array}$ \\
\hline
\end{tabular}

Abbreviations: ESKD, end-stage kidney disease; MCLKD, medically complex living kidney donors; TS, transplant surgeons.

with DM for 1 year, even if it was controlled with monotherapy or diet.

Our study's findings regarding the acceptance rate of donors with stage I obesity, decline rate of obese candidates with FH of DM, exclusion rate of candidates based only on FG values, and the rate of pursing GTT for elevated $1 \mathrm{HbA} 1 \mathrm{C}$ are comparable to the findings of Garg et al in $2017 .^{15}$

While programs are becoming less restrictive about accepting donors with BMI more than 35 or $40,{ }^{15}$ bariatric surgery prior to living donor nephrectomy may be a solution to expand the living donor kidney pool. In a study of 22 donors who had bariatric surgery, donors had waited 0.7 to 22 years prior to laparoscopic living donor nephrectomy. Eighteen donors would have been excluded from donation prior to bariatric surgery, as their BMI was more than 40 and 17 reached a BMI of less than 35 after bariatric surgery. ${ }^{38}$ Nguyen et al advocated that bariatric surgery should be considered in preparation for donation in morbidly obese candidates, since this can positively alter their candidacy without significantly impacting the subsequent living donor nephrectomy and early outcomes. ${ }^{38}$ In another study, bariatric surgery prior to living kidney donation by an obese donor was also found to be cost-effective (with a ratio of $3.64 \mathrm{vs}$. a kidney transplant from a deceased donor). ${ }^{39}$ In our survey, donor candidates with resolved DM postbariatric surgery due to weight reduction could be considered for donation as soon as surgery was possible or when their BMI reduced to less than 30 . However, more respondents (44\%) preferred to have a waiting period postbariatric surgery to ensure no relapse.

According to our survey, potential donors with isolated obesity, IFG, mildly abnormal HgbA1C, or history of bariatric surgery were considered for kidney donation by many respondents. However, donors with more than one abnormality, especially young donors, were not likely to be considered for donation.

Donor candidates with hypertension that can be controlled to systolic $\mathrm{BP}<140 \mathrm{~mm} \mathrm{Hg}$ and diastolic $\mathrm{BP}<90 \mathrm{~mm}$ $\mathrm{Hg}$ using one or two antihypertensive agents, with no evidence of target organ damage, may be acceptable for donation. ${ }^{8,40}$ In $2017,65 \%$ of the renal transplant programs in the United States accepted donors with monotherapy-controlled hypertension, and only $10 \%$ of programs considered donor candidates on two antihypertensive medications. ${ }^{32}$ The decision to approve donor candidates with hypertension should be individualized, based on the health profile of the donor and in consideration of the acceptable risk threshold of the transplant program. ${ }^{8,40,41}$

Female LKDs are 2.4 times more likely to develop gestational hypertension or preeclampsia (95\% CI 1.2 to 5.0 ) compared with healthy nondonors. ${ }^{42}$ However, most donor pregnancies remain uncomplicated with no significant neonatal or maternal gestational complications. ${ }^{42}$ Thus, females of childbearing age should be counseled about these risks before donation. In our survey, we found that the majority of respondents would allow women with mild and controlled hypertension to proceed with living kidney donation only if they had no future plans for pregnancy.

In addition, normotensive LKDs may have a $5-\mathrm{mm} \mathrm{Hg}$ greater increase in BP than the increase anticipated with normal aging. ${ }^{43}$ After kidney donation, up to $3 \%, 4 \%, 10 \%$, and $51 \%$ of LKDs may develop hypertension at $2,5,10$, and 40 years, respectively. ${ }^{44,45}$ Donors who are male, obese, and who have a first-degree relationship are at higher risk. $^{44}$ The risk of developing hypertension after donation is increased by a HR $(1.19 ; 95 \% \mathrm{CI}, 1.01$ to $1.41 ; p=0.04) .{ }^{46}$ 
Hypertension after donation may contribute to the increased risk of ESKD, cardiovascular, and all-cause mortality of donors compared with healthy nondonors. ${ }^{4,47}$ The risk is also higher in healthy young donors than healthy older candidates. $^{48}$

Up to $10 \%$ of donors have white coat hypertension (elevated $\mathrm{BP}$ readings measured in the clinic but normal $\mathrm{BP}$ by ABPM). ${ }^{8,40,41}$ White coat hypertension and abnormal ABPM patterns such as nondipping BP at night are potentially associated with increased cardiovascular risk. ${ }^{49,50}$ Neither white coat syndrome nor abnormal patterns of ABPM per se are contraindications for kidney donation. However, they are considered as indicators of increased risk, especially in young donors who are already at higher risk than their older counterparts.

The donor-recipient age discrepancy comprises another dilemma in deciding kidney transplantation. A very young age (18 to 21 years) is viewed as a relative contraindication for kidney donation by many programs (23\% of programs in the United States). ${ }^{16,51,52}$

Programs are more restrictive in applying exclusion criteria for younger donors than older donors when it comes to renal risk factors. ${ }^{48}$ This is because young LKDs are expected to have more years of exposure to renal risk factors postkidney donation than older donors. ${ }^{4,47,48}$ Young female donors of childbearing age are also prone to the potential risks associated with future pregnancies, including gestational hypertension or preeclampsia. ${ }^{42}$

In our survey, the majority of respondents would accept a very young potential donor, especially if they were males. However, up to $20 \%$ would decline them regardless of the donor's gender. Plans for future pregnancies were also an important factor when counseling a potential female donor of childbearing age.

In contrast, most transplant programs in the United States have no upper age limit for accepting donors, ${ }^{32}$ but they require careful assessment of risk factors, especially for donors over 65 years, since these grafts can be associated with poorer outcomes. ${ }^{53,54}$ The donor and recipient should be advised that grafts from LKD who are 65 to 69 years have similar (but not better) graft survival of standard criteria donor (SCD) and grafts from donors aged more than 70 years have worse graft survival of SCD. ${ }^{53}$ In our survey, the majority of respondents would accept a donor over 65 years to donate to an 18-year-old recipient with expected prolonged waiting time for a deceased donor.

LKD transplantation in a recipient over 70 years is also challenging, especially if the donor is very young. Renal transplantation decreases all-cause mortality in elderly patients when compared with dialysis. ${ }^{54}$ While graft survival may be similar, older transplant recipients have a higher mortality risk than their younger counterparts. ${ }^{55}$ LKD transplantation for recipients over 70 years is associated with lower mortality than observed with SCD or expanded criteria donor (ECD). ${ }^{53}$ However, higher age of the recipient (e.g., 6070 years vs. $70-80$ or over 80 years) is a strong, independent risk factor for death after kidney transplantation. ${ }^{53}$ Criteria for and appropriateness of renal transplantation in elderly patients with ESKD should be based on frailty score, comorbidities, and potential life expectancy. ${ }^{56}$ In our survey, we found that the opinions about allowing a very young donor to donate to a very old recipient were divided in the transplant community.

The acceptance of LKDs with FH of kidney disease of unknown etiology is challenging. Up to $40 \%$ of LKDs are close biological relatives to the recipients. ${ }^{57}$ Many of those biologically related donors are also in their 30 s and 40 s. This makes it more difficult to predict future risk of kidney disease, especially if the cause of kidney failure of the potential recipient is unknown and genetic etiology cannot be ruled out. Genetic screening for a specific disorder such as polycystic kidney disease, Alport disease, or atypical hemolytic uremic syndrome is typically guided by clinical indications. There is uncertainty about the interpretation of APOL1 status and its renal risk, and there is no consensus about its utility of the evaluation of potential LKD. ${ }^{16,58-61}$

Comprehensive genetic screening of LKDs with $\mathrm{FH}$ of chronic kidney disease with unknown etiology has several limitations. First, the majority of kidney diseases are polygenic or secondary to known risk factors of chronic kidney disease such as diabetes or hypertension. ${ }^{62}$ Second, not all genetic variants are identified by targeted next-generation sequencing panels (or whole-exome sequencing). ${ }^{62}$ Finally, significant variants unrelated to the phenotype (unsolicited but medically significant discoveries) may be identified. ${ }^{62-64}$

In one recent study, family members of patients with ESKD from "nonhereditary" causes of ESKD had increased relative risk of ESKD when compared with those with no $\mathrm{FH}$ of renal disease (risk ratio [RR]: 3.7; 95\%, CI: 3.1 to 4.4). The study identified glomerular disease, interstitial disease, diabetic nephropathy, and hypertensive nephrosclerosis among "nonhereditary" causes of ESKD. ${ }^{65}$

In our survey, FH of kidney disease of unknown etiology was a significant determining factor to decline young donors.

-Table 6 summaries the findings of the survey.

There are several strengths and limitations in our study. The survey was answered by 239 respondents, including 100 nephrologists and 139 TSs, from 29 countries through three major international transplant societies (AST, ASTS, and ERAEDTA). The number of participants in our survey was much larger than that of previous surveys distributed through UNOS in 2007 and 2017 (for instance, the survey distributed through UNOS in 2017 had 72 responses, with 44 medical directors and 28 surgical directors). This was due to the multinational nature of our study and the fact that we did not restrict participation to the directors of the transplant units.

Our survey also covered several challenging areas that had not been addressed by previous guidelines or surveys. Potential living donors and recipients are typically assessed by separate health care teams to avoid any conflict of interest (75). Our survey was unique in exploring the difference between nephrologists and TS, although the main objective of our survey was exploring the opinions of nephrologists and TS as a unified group, since the decisions of accepting MCLKDs are typically addressed through a multidisciplinary approach. 
Table 6 Summary of the survey findings

1- The vast majority (75\%) of the respondents would allow an obese potential donor (BMI 30-35) to donate if the donor started to demonstrate some weight loss or intention to lose weight.

2- Only $45 \%$ of the respondents will allow an obese potential donor with a strong FH of DM to donate if there were no alternative donor without further requirements or she/he intends to lose weight.

3- Stage Il obese potential donors have to demonstrate some weight loss or even reach a BMI of 30 before being considered for kidney donation by most of the respondents.

4- Potential donors with obesity and IFG can donate only if impaired BS is resolved due to weight reduction according to half of the respondents.

5- More than half of the respondents request GTT to verify isolated and mildly elevated A1C or IFG in a potential donor before deciding on candidacy.

6- Most respondents would decline a middle-age donor candidate with diabetes for one year even if it was controlled with just monotherapy or diet.

7- Donation candidates with resolved DM postbariatric surgery due to weight reduction can be considered for donation as soon as surgery is possible or when the BMI reduces to less than 30. Other respondents preferred a waiting period postbariatric surgery to ensure no relapse.

8- Potential donors with isolated obesity, IFG, mildly abnormal HgbA1C or history of bariatric surgery are still considered for kidney donation by many respondents. However, donors with more than one abnormality especially at a young are likely not to be considered for donation.

9- A majority of respondents would allow women with mild and controlled hypertension to proceed with living donation only if they have no future plans for pregnancy.

10- Plan of future pregnancies is an important factor when counseling a potential female donor of childbearing age.

11- Most respondents are reluctant to accept very young male donors if they show higher risk of developing hypertension in the future.

12- The majority of respondents will allow very young donor candidates to donate especially in males. However up to $20 \%$ will decline this donation regardless of the gender of the donor.

13- The transplant community is divided about allowing a very young donor to donate to a very old recipient.

14- The majority will accept a > 65-year-old donor to donate to an 18-year-old recipient with expected prolonged waiting time for a deceased donor.

15- FH of kidney disease of unknown etiology is an important factor to decline young donors.

Abbreviations: BMI, body mass index; DM, diabetes mellitus; FH, family history; GTT, glucose tolerance test; IFG, impaired fasting glucose.

One of the limitations of our study is that respondents practicing in the United States were overrepresented $65 \%$ of the total respondents were from the United States, whereas only $25 \%$ of worldwide renal transplantations occurred in the United States in 2019) (76-77). This is because we did not have official access to directly approach the renal transplant units globally, and the respondents were reached through the international societies, many of which did not have any means to distribute the external studies.

Other potential limitations of the survey are the unknown response rate of EDTA members and the fact that we did not account for multiple replies from the same unit. Again, this was due to the distribution of our survey through transplant societies by email and web posting. These do not critically affect the validity of our study, since it was designed to explore the general opinions of the transplant providers in the international community and not the formal policies of the transplant units. Other assuring factors include the high completion rate of $99 \%$ and the comparisons made between our results and previous surveys, as outlined throughout the discussion.

\section{Conclusion}

This survey highlights the practice patterns of the acceptance of MCLKDs among the international community. In the absence of clear guidelines, this survey provides additional information to counsel kidney donors with obesity, hypertension, FH of kidney disease, and donor-recipient age discrepancy.

\section{Disclosure}

The authors declare no conflicts of interest.

Financial Support

None.

Acknowledgment

We would like to thank AST and ASTS for distributing this external survey. We also would like to thank ERA-EDTA for endorsing and distributing this survey for the promotion of education, without responsibility for the content of the survey. Finally, we would like to thank Dr. Osun Kwon for her editorial review and Dr. A. A. Awad for performing the statistical analysis. 


\section{References}

1 Maggiore U, Cravedi P. The marginal kidney donor. Curr Opin Organ Transplant 2014;19(04):372-380

2 Reese PP, Feldman HI, McBride MA, Anderson K, Asch DA, Bloom RD. Substantial variation in the acceptance of medically complex live kidney donors across US renal transplant centers. Am J Transplant 2008;8(10):2062-2070

3 Maggiore U, Budde K, Heemann U, et al; ERA-EDTA DESCARTES working group. Long-term risks of kidney living donation: review and position paper by the ERA-EDTA DESCARTES working group. Nephrol Dial Transplant 2017;32(02):216-223

4 Muzaale AD, Massie AB, Wang M-C, et al. Risk of end-stage renal disease following live kidney donation. JAMA 2014;311(06): 579-586

5 Halverson CME, Wang JY, Poulson M, Karlin J, CrowleyMatoka M, Ross LF. Living kidney donors who develop kidney failure: excerpts of their thoughts. Am J Nephrol 2016;43 (06):389-396

6 Kiberd BA, Tennankore KK. Lifetime risks of kidney donation: a medical decision analysis. BMJ Open 2017;7(08):e016490

7 Abramowicz D, Cochat P, et al. The European Renal Best Practice Transplantation guideline development g, Guideline. Nephrol Dial Transplant 2013;28(Suppl 2):ii1-ii71

8 Lentine KL, Kasiske BL, Levey AS, et al. KDIGO Clinical Practice Guideline on the Evaluation and Care of Living Kidney Donors. Transplantation 2017;101(8S, Suppl 1)S1-S109

9 Abramowicz D, Cochat P, Claas FHJ, et al. European Renal Best Practice Guideline on kidney donor and recipient evaluation and perioperative care. Nephrol Dial Transplant 2015;30(11): 1790-1797

10 ESRD Risk Tool for Kidney Donor Candidates Accessed September 16, 2021 at: http://wwwtransplantmodelscom/esrdrisk/

11 Grams ME, Sang Y, Levey AS, et al; Chronic Kidney Disease Prognosis Consortium. Kidney-failure risk projection for the living kidney-donor candidate. N Engl J Med 2016;374(05): 411-421

12 Figurek A, Luyckx VA, Mueller TF. A systematic review of renal functional reserve in adult living kidney donors. Kidney Int Rep 2020;5(04):448-458

13 Cantarelli C, Cravedi P. Criteria for living donation from marginal donors: one, no one, and one hundred thousand. Nephron 2019; 142(03):227-232

14 Lafranca JA, Spoon EQW, van de Wetering J, IJzermans JNM, Dor FJMF. Attitudes among transplant professionals regarding shifting paradigms in eligibility criteria for live kidney donation. PLoS One 2017;12(07):e0181846

15 Garg N, Lentine KL, Inker LA, et al. Metabolic, cardiovascular, and substance use evaluation of living kidney donor candidates: US practices in 2017. Am J Transplant 2020;20(12): $3390-3400$

16 Garg AX, Levey AS, Kasiske BL, Cheung M, Lentine KLKDIGO Clinical Practice Guideline on the Evaluation and Care of Living Kidney Donors Work Group and Evidence Review Team. Application of the 2017 KDIGO Guideline for the Evaluation and Care of Living Kidney Donors to Clinical Practice. Clin J Am Soc Nephrol 2020;15(06):896-905

17 Prevention CfDC. Defining Adult Overweight and Obesity. Accessed April 14, 2020 at: https://wwwcdcgov/obesity/adult/ defininghtml

18 Europe WROf. Body mass index. Accessed September 16, 2021 at: http://wwweurowhoint/en/health-topics/disease-prevention/nutrition/a-healthy-lifestyle/body-mass-index-bmi

19 Ganz ML, Wintfeld N, Li Q, Alas V, Langer J, Hammer M. The association of body mass index with the risk of type 2 diabetes: a case-control study nested in an electronic health records system in the United States. Diabetol Metab Syndr 2014;6(01):50
20 Gray N, Picone G, Sloan F, Yashkin A. Relation between BMI and diabetes mellitus and its complications among US older adults. South Med J 2015;108(01):29-36

21 Praga M, Hernández E, Herrero JC, et al. Influence of obesity on the appearance of proteinuria and renal insufficiency after unilateral nephrectomy. Kidney Int 2000;58(05):2111-2118

22 Hsu CY, McCulloch CE, Iribarren C, Darbinian J, Go AS. Body mass index and risk for end-stage renal disease. Ann Intern Med 2006; 144(01):21-28

23 Taner T, Iqbal CW, Textor SC, Stegall MD, Ishitani MB. Compensatory hypertrophy of the remaining kidney in medically complex living kidney donors over the long term. Transplantation 2015;99 (03):555-559

24 Tavakol MM, Vincenti FG, Assadi H, et al. Long-term renal function and cardiovascular disease risk in obese kidney donors. Clin J Am Soc Nephrol 2009;4(07):1230-1238

25 Taler S. The Obese Kidney Donor Living Donor Community of Practice of AST. Accessed September 16, 2021 at: https://www. myast.org/sites/default/files/pdf/Chapter\%205\%20\%20The\% 20obese\%20donor.pdf

26 Scott RA, Langenberg C, Sharp SJ, et al; InterAct Consortium. The link between family history and risk of type 2 diabetes is not explained by anthropometric, lifestyle or genetic risk factors: the EPIC-InterAct study. Diabetologia 2013;56(01):60-69

27 Garg N, Lentine KL, Inker LA, et al. The kidney evaluation of living kidney donor candidates: US practices in 2017. Am J Transplant 2020

28 Jacobs SC, Cho E, Dunkin BJ, et al. Laparoscopic nephrectomy in the markedly obese living renal donor. Urology 2000;56(06): 926-929

29 Lentine KL, Lam NN, Axelrod D, et al. Perioperative complications after living kidney donation: a national study. Am J Transplant 2016;16(06):1848-1857

30 Reese PP, Feldman HI, Asch DA, Thomasson A, Shults J, Bloom RD. Short-term outcomes for obese live kidney donors and their recipients. Transplantation 2009;88(05):662-671

31 Heimbach JK, Taler SJ, Prieto M, et al. Obesity in living kidney donors: clinical characteristics and outcomes in the era of laparoscopic donor nephrectomy. Am J Transplant 2005;5(05): 1057-1064

32 Mandelbrot DA, Pavlakis M, Danovitch GM, et al. The medical evaluation of living kidney donors: a survey of US transplant centers. Am J Transplant 2007;7(10):2333-2343

33 American Diabetes Association. Diagnosis and classification of diabetes mellitus. Diabetes Care 2013;36(Suppl 1):S67-S74

34 Kumar R, Nandhini LP, Kamalanathan S, Sahoo J, Vivekanadan M. Evidence for current diagnostic criteria of diabetes mellitus. World J Diabetes 2016;7(17):396-405

35 Kim D-L, Kim S-D, Kim SK, Park S, Song K-H. Is an oral glucose tolerance test still valid for diagnosing diabetes mellitus? Diabetes Metab J 2016;40(02):118-128

36 Lee J, Lee YA, Kim JH, Lee SY, Shin CH, Yang SW. Discrepancies between glycosylated hemoglobin and fasting plasma glucose for diagnosing impaired fasting glucose and diabetes mellitus in Korean youth and young adults. Diabetes Metab J 2019;43(02): 174-182

37 Yu EYT, Wong CKH, Ho SY, Wong SYS, Lam CLK. Can HbA1c replace OGTT for the diagnosis of diabetes mellitus among Chinese patients with impaired fasting glucose? Fam Pract 2015;32(06): 631-638

38 Nguyen MJP, Carpenter D, Tadros J, et al. Bariatric surgery prior to living donor nephrectomy: a solution to expand the living donor kidney pool - a retrospective study. Transpl Int 2019;32(07): 702-709

39 Brooks J, Mitro G, Deleonibus A, et al. Bariatric surgery for obese live kidney donors: an analysis of risks and benefits. Integr Obes Diabetes 2017 (e-pub ahead of print). Doi: 10.15761/IOD.1000186 
40 Andrews PA, Burnapp L. British Transplantation Society / Renal Association UK Guidelines for Living Donor Kidney Transplantation 2018: Summary of Updated Guidance. Transplantation 2018; 102(07): 307

41 Ierino F, Boudville N, Kanellis JCARI. The CARI guidelines. Donors at risk: hypertension. Nephrology (Carlton) 2010;15(Suppl 1):S114-S120

42 Garg AX, Nevis IF, McArthur E, et al; DONOR Network. Gestational hypertension and preeclampsia in living kidney donors. $\mathrm{N}$ Engl J Med 2015;372(02):124-133

43 Boudville N, Prasad GV, Knoll G, et al; Donor Nephrectomy Outcomes Research (DONOR) Network. Meta-analysis: risk for hypertension in living kidney donors. Ann Intern Med 2006;145 (03):185-196

44 Holscher CM, Bae S, Thomas AG, et al. Early hypertension and diabetes after living kidney donation: a national cohort study. Transplantation 2019;103(06):1216-1223

45 Sanchez OA, Ferrara LK, Rein S, Berglund D, Matas AJ, Ibrahim HN. Hypertension after kidney donation: incidence, predictors, and correlates. Am J Transplant 2018;18(10):2534-2543

46 Holscher CM, Haugen CE, Jackson KR, et al. Self-reported incident hypertension and long-term kidney function in living kidney donors compared with healthy nondonors. Clin J Am Soc Nephrol 2019;14(10):1493-1499

47 Mjøen G, Hallan S, Hartmann A, et al. Long-term risks for kidney donors. Kidney Int 2014;86(01):162-167

48 Steiner RW, Ix JH, Rifkin DE, Gert B. Estimating risks of de novo kidney diseases after living kidney donation. Am J Transplant 2014;14(03):538-544

49 Pioli MR, Ritter AM, de Faria AP, Modolo R. White coat syndrome and its variations: differences and clinical impact. Integr Blood Press Control 2018;11:73-79

50 Staessen JA, Thijs L, Fagard R, et al; Systolic Hypertension in Europe Trial Investigators. Predicting cardiovascular risk using conventional vs ambulatory blood pressure in older patients with systolic hypertension. JAMA 1999;282(06):539-546

51 Campbell M, Wright L, Greenberg RA, Grant D. How young is too young to be a living donor? Am J Transplant 2013;13(07):1643-1649

52 Kher A, Mandelbrot DA. The living kidney donor evaluation: focus on renal issues. Clin J Am Soc Nephrol 2012;7(02):366-371
53 Karim A, Farrugia D, Cheshire J, et al. Recipient age and risk for mortality after kidney transplantation in England. Transplantation 2014;97(08):832-838

54 Huang E, Segev DL, Rabb H. Kidney transplantation in the elderly. Semin Nephrol 2009;29(06):621-635

55 Calabuig AS, Martínez EG, Berga JK, et al. Kidney transplantation in recipients older than 70 years old: a good option for our patients. Transplantation 2018;102:S468

56 Segall L, Nistor I, Pascual J, et al. Criteria for and appropriateness of renal transplantation in elderly patients with end-stage renal disease: a literature review and position statement on behalf of the European Renal Association-European Dialysis and Transplant Association Descartes Working Group and European Renal Best Practice. Transplantation 2016;100(10):e55-e65

57 Hart A, Smith JM, Skeans MA, et al. Kidney. Am J Transplant 2016; 16(Suppl 2):11-46

58 Nadkarni GN, Gignoux CR, Sorokin EP, et al. Worldwide Frequencies of APOL1 Renal Risk Variants. N Engl J Med 2018;379(26): 2571-2572

59 Parsa A, Kao WH, Xie D, et al; AASK Study Investigators; CRIC Study Investigators. APOL1 risk variants, race, and progression of chronic kidney disease. N Engl J Med 2013;369(23):2183-2196

60 Devuyst O. Genetic variants and risk of chronic kidney disease. Perit Dial Int 2014;34(02):150-150

61 Mohan S, Iltis AS, Sawinski D, DuBois JM. APOL1 genetic testing in living kidney transplant donors. Am J Kidney Dis 2019;74(04): 538-543

62 Thomas CP, Mansilla MA, Sompallae R, et al. Screening of living kidney donors for genetic diseases using a comprehensive genetic testing strategy. Am J Transplant 2017;17(02):401-410

63 Corredor Z, Filho MIDS, Rodríguez-Ribera L, et al. Genetic variants associated with chronic kidney disease in a Spanish population. Sci Rep 2020;10(01):144

64 Cañadas-Garre M, Anderson K, Cappa R, et al. Genetic susceptibility to chronic kidney disease - Some more pieces for the heritability puzzle. Front Genet 2019;10(453):453

65 Skrunes R, Svarstad E, Reisæter AV, Vikse BE. Familial clustering of ESRD in the Norwegian population. Clin J Am Soc Nephrol 2014;9 (10):1692-1700 